\title{
Ganglioneuroma coincidente con una extrusión discal lumbar
}

\author{
J.V. Martínez-Quiñones; F. Consolini; J. Aso-Escario; M. Domínguez-Páez* y R. Arregui
}

Servicio de Neurocirugía. Hospital MAZ. Zaragoza. *Servicio de Neurocirugía. Hospital Regional Universitario Carlos Haya. Málaga.

\section{Resumen}

Introducción. Los ganglioneuromas o gangliomas son tumores de los ganglios simpáticos que contienen células de la cresta neural, pudiendo aparecer en cualquier parte del organismo. Son generalmente benignos, más frecuente entre los 10 y los 40 años, pueden secretar hormonas y, en ocasiones, asociarse a trastornos genéticos, como la neurofibromatosis tipo I.

Objetivo. Revisar la literatura científica relacionada con el tema y presentar un caso tratado en nuestro servicio.

Discusión. La sintomatología de estas lesiones depende de su ubicación y de las sustancias vasoactivas que puedan secretar. A pesar de que son tumores benignos en su mayoría, en ocasiones pueden metastatizar. Las pruebas de imagen y de laboratorio son inespecíficas, por lo que el diagnóstico definitivo es anatomopatológico. En los casos sintomáticos el tratamiento de elección es la resección quirúrgica.

Conclusión. La asociación de un ganglioneuroma y de una hernia discal lumbar es algo excepcional. El tratamiento requiere su resección quirúrgica.

PALABRAS CLAVE. Ganglioneuroma. Ganglioma. Hernia discal.

Ganglioneuroma coinciding with a lumbar disk herniation

\section{Summary}

Introduction. Ganglioneuromas or gangliomas are tumours of the sympathetic ganglia that contain cells of the neural crest, so they can appear in all body localizations. They are generally benign, more frequent between 10 and 40 years, may secrete hormones and, sometimes, Neurofibromatosis type I and other genetic disorders can be associated.

Recibido: 25-03-10. Aceptado: 18-07-11
Objective. To review the scientific literature related to the topic and to present a case treated in our service.

Discussion. The symptoms depend on location and vasoactive secreted hormones. In spite of that, they are generally benign tumours, although sometimes they can spread out. Since laboratory and image test are of limited usefulness, the conclusive diagnosis is anatomopathologic. In symptomatic patients the best procedure is surgical revoming.

Conclusión. Ganglioneuroma and disk herniation association constitute an excepcional disorder. Its treatment implies surgery resection.

KEY WORDS. Ganglioneuroma. Ganglioma. Disk herniation.

Introducción

La concurrencia de un ganglioneuroma con una hernia discal lumbar es un suceso muy infrecuente. En la revisión de la literatura realizada sólo se ha encontrado un caso publicado $^{18}$. Dado lo peculiar de la asociación, presentamos un caso clínico en el que acontece dicho fenómeno.

\section{Caso Clínico}

Varón de 56 años edad de raza caucásica, trabajador de la construcción, que llegó a nuestra consulta por una lumbociática derecha postesfuerzo de 24 horas de evolución. En la exploración se apreciaba una radiculopatía de predominio motor S1 derecha, caracterizada por una menor fuerza en la flexión plantar y dificultad para caminar de puntillas. El cuadro doloroso resultó muy intenso e incapacitante, con mala respuesta al tratamiento convencional. El estudio de RM permitió apreciar la existencia de una hernia discal extruida a nivel del segmento L5-S1, con migración de un fragmento a nivel del receso lateral derecho, sin que se sospechara nada anómalo concomitante. Se asociaban sendas discopatías degenerativas L3-L4 y L4L5. (Figura 1, A y B). El estudio neurofisiológico descartó la existencia de actividad denervativa radicular aguda. 


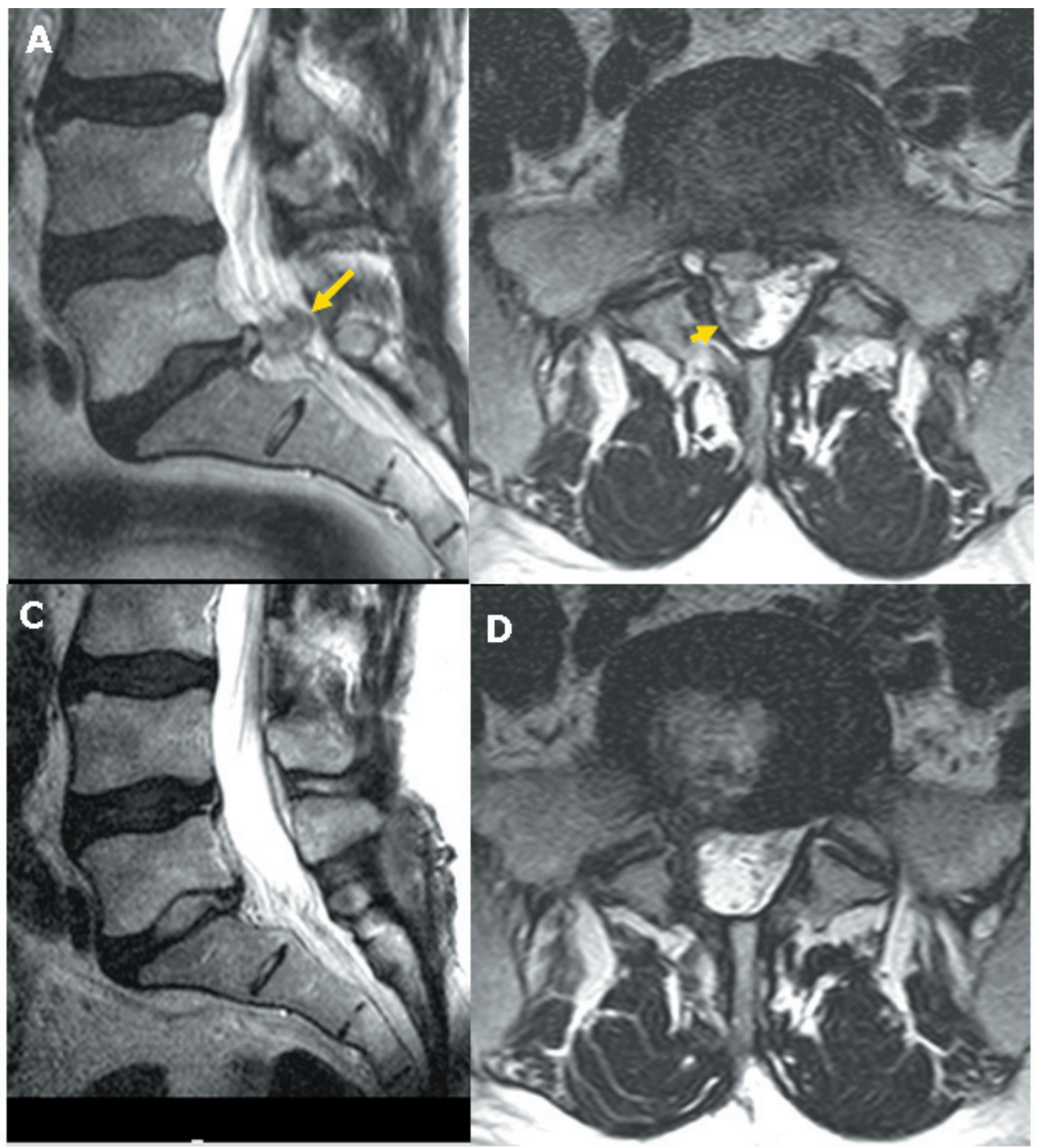

Figura 1. Estudio de RM pre- $(A, B)$ y post- quirúrgico (C, D). La flecha señala lo que se interpretó como secuestro discal.

Debido a la persistencia de la clínica deficitaria del paciente, se decidió llevar a cabo de forma precoz, un mes desde su llegada a consultas, una descompresión radicular mediante microdiscectomía convencional. Durante la misma, se identificó una raíz S1 desplazada dorso-lateralmente por un fragmento discal extruido que se extrajo completamente, permitiendo identificar una masa extradural, fusiforme, bien definida, de consistencia elástica, coloración blanco-grisácea y de aproximadamente $1,5 \mathrm{~cm}$ de longitud en su diámetro mayor, que se encontraba íntimamente adherida a la raíz en sus extremos cráneocaudal, dando la sensación de que formaba parte de la misma. Tras una disección minuciosa de dicha estructura se decidió incidirla y, tras comprobar que no se producía salida de LCR, se procedió a su exéresis completa. Una vez resecada, el contenido parecía más fibrótico que nervioso. Posteriormente se completó la microdiscectomía. El paciente toleró bien el acto quirúrgico, no apreciándose déficit motor añadido en el postoperatorio inmediato, sin embargo, refirió sensaciones disestésicas quemantes en la extremidad inferior derecha que se controlaron con pregabalina. La RM postoperatoria no mostró complicaciones (Figura 1, C y D). El estudio anatomopatológico determinó que la masa resecada en íntima relación a la raíz nerviosa correspondía a fragmentos de tejido conectivo-fibroso que englobaban segmentos de fascículos nerviosos dispuestos 


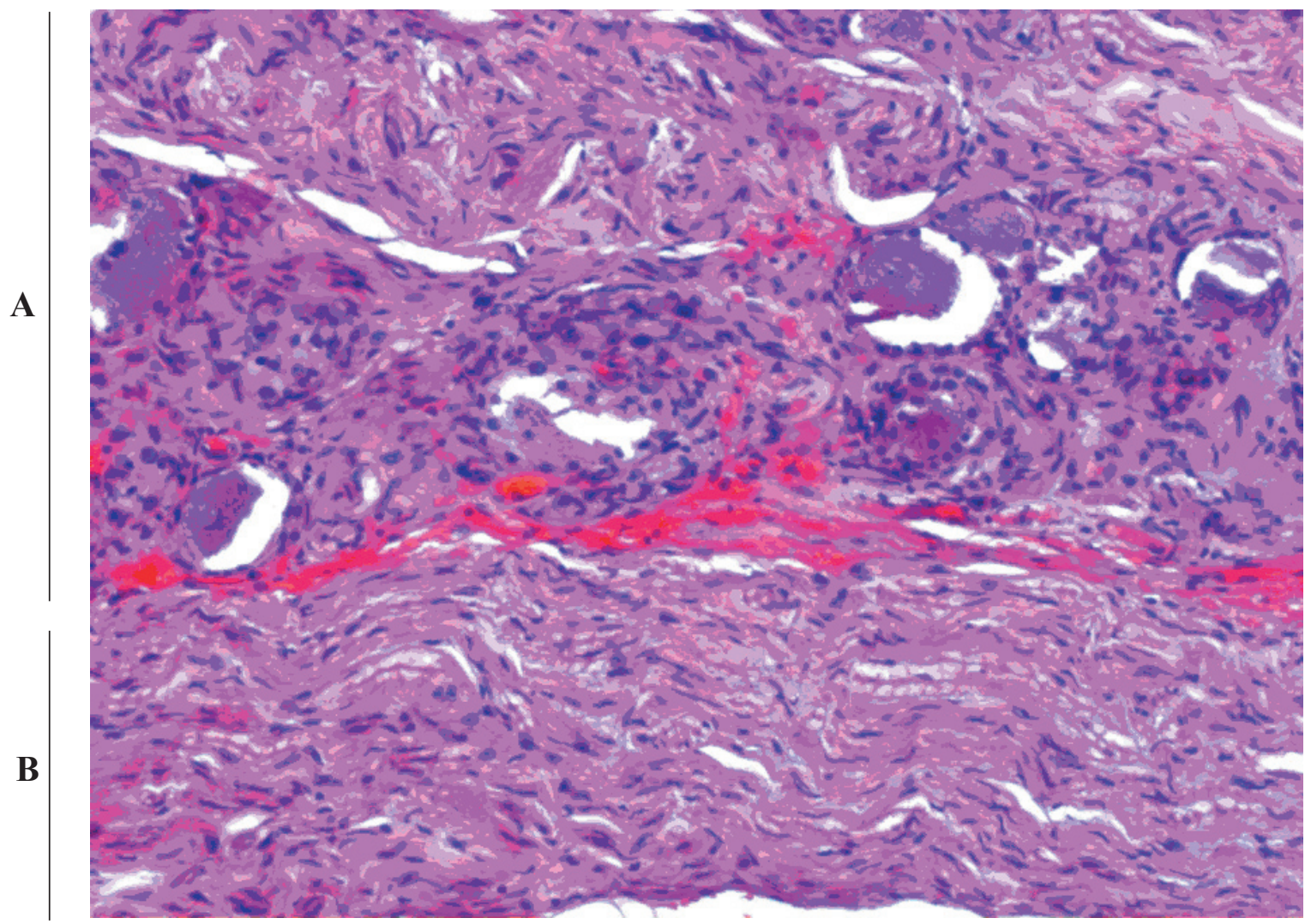

Figura 2. A. Vasos sanguíneos, fondo neuroide y abundantes células ganglionares; B. Tronco nervioso. Histoarquitectura compatible con el diagnóstico de ganglioneuroma.

de forma ordenada junto a estroma schwanniano, así como células ganglionares maduras, todo ello compatible con el diagnóstico de ganglioneuroma (Figura 2). Tres meses después de la intervención el paciente fue dado de alta médica, reintegrándose a su vida laboral habitual.

\section{Discusión}

Los ganglioneuromas son tumores benignos que derivan de la cresta neural por proliferación de células maduras de Schwann y fibroblastos. La edad típica de presentación oscila entre los 10 y los 40 años. Se ha descrito su asociación con trastornos genéticos, como la neurofibromatosis tipo $I^{3,13,16}$. Suelen evolucionar de forma asintomática, descubriéndose tras un examen rutinario o por un tratamiento por otra afección. Si producen síntomas, estos dependerán de la ubicación y del tipo de hormonas que secreten, aunque suelen resultar hormonalmente silentes ${ }^{5}$. En ocasiones pueden secretar polipéptidos intestinalmente vasoactivos, induciendo entonces un síndrome caracterizado por diarrea, hipopotasemia y aclorhidria ${ }^{28}$, o testosterona, lo que conlleva virilizaciones ${ }^{7}$. Se han descrito ubicaciones en cualquier zona del organismo: supratentoriales ${ }^{3}$, cerebelosas $^{15}$, raquídeas $^{4,8,10,12,13,24}$, mediastínicas ${ }^{27}$, retroperitoneales ${ }^{22}$, intestinales ${ }^{11}$ o cutáneas ${ }^{6}$. Cuando su localización es cercana a la medula espinal es frecuente la compresión de la misma o la tracción radicular, pudiendo causar dolor, pérdida de fuerza o trastornos sensoriales en dependencia del nivel mielo-radicular afecto.

En cuanto al diagnóstico, son lesiones que ni ofrecen imágenes típicas en los estudios radiológicos, ni datos bioquímicos específicos. En RM pueden tomar la apariencia de "dumbbell tumors" o tumores en "reloj de arena", tumores intradurales, extradurales o paravertebrales 1,9,20,21,26. El diagnóstico diferencial de los tumores en "reloj de arena" incluye como lesiones más habituales el schwannoma, neurofibroma y ependimoma mixopapilar ${ }^{19}$; la migración de una hernia discal lumbar puede simular un "dumbbell tumor" de forma excepcional ${ }^{25}$. En nuestro caso el tumor se presentó como una masa extradural asociada a una hernia discal extruida. Hasta el momento sólo existe publicado un caso más de ganglioneuroma asociado a una extrusión discal lumbar ${ }^{18}$. En los dos casos reportados en $1956^{8}$ la lumbociatalgia ocasionada por el ganglioneuroma no se asociaba a material discal extruido. Dada la inespecificidad de las pruebas complementarias el diagnóstico definitivo se consigue tras el análisis anatomopatológico. Macroscópicamente son lesiones firmes y encapsuladas que se disponen a lo 
largo de los nervios centrales y/o periféricos, englobando a veces porciones de fibras nerviosas. Microscópicamente es característica la presencia de abundante tejido conjuntivofibrótico junto a células nerviosas mielínicas, amielínicas y ganglionares simpáticas ${ }^{7}$. Es típico encontrar en estas células ganglionares signos de madurez: citoplasma abundante, núcleos vesiculosos y nucleolo evidente ${ }^{23}$. La presencia de estroma schwanniano se asocia a un pronóstico más favorable $^{14}$. La inmunohistoquímica positiva para sinaptofisina, neurofilamentos y proteína fibrilar glial ácida apoyan el diagnóstico histológico ${ }^{3}$.

A pesar de que han sido reportados casos de comportamiento agresivo y/o metástasis a distancia, la evolución de los ganglioneuromas suele ser benigna ${ }^{17}$, por lo tanto el tratamiento quirúrgico está especialmente indicado en aquellos tumores que provocan síntomas por compresión. Aquellos con apariencia de "reloj de arena" requieren un doble abordaje, siendo prioritaria la descompresión vía posterior de las estructuras nerviosas ${ }^{2}$.

\section{Conclusión}

Los ganglioneuromas son tumores que pueden ubicarse en cualquier región del organismo, simulando afecciones propias de las zonas en las que asientan. Su coincidencia con una hernia discal es altamente infrecuente. El tratamiento quirúrgico está especialmente indicado en las lesiones sintomáticas, siendo recomendable la resección completa dada la posibilidad de comportamiento agresivo.

\section{Bibliografía}

1. Adrien, C., Kiselman, R., Montagne, J.P.: Iconographic rubric. Lumbar ganglioneuroma. Arch Fr Pediatr 1991; 48: 275-276.

2. Belzberg, A. J.; Dorsi, M. J.: Neoplasms of peripheral nerves. En: Fessler, R. G.; Sekhar, L. Atlas of neurosurgical techniques: spine and peripheral nerves. New York; Thieme, 2006; pp: 879-987.

3. Carrasco-González, A., Lafuente-Sánchez, J.V., Pomposo-Gaztelu, I., et al.: Neuronal tumors: gangliocytoma. Rev Neurol 2008; 46: 155-159.

4. Cerullo, G., Marrelli, D., Rampone, B., et al.: Presacral ganglioneuroma: a case report and review of literature. World J Gastroenterol 2007; 13: 2129-2131.

5.Erem, C., Ucunzo, O., Nuhoglu, I., et al.: Adrenal ganglioneuroma: report of a new case. Endocrine 2009; 35 : 293-296.

6. Furmanczyk, P.S., Hughes, S.R., Walsh, J.S., Bass, J., McFarlane, J.R., Argenyi, Z.B.: Cutaneous ganglioneuroma associated with overlying hyperkeratotic epidermal changes: a report of 2 cases. Am J Dermatopathol 2008; 30: 600-603.

7. Gaisa, N.T., Klöppel, G., Brehmer, B., et al.: Virilizing adrenal ganglioneuroma: A rare differential diagnosis in testosterone secreting adrenal tumours. Pathologe 2009; 30: 407-410.

8. Goidanich, I.F., Venturi, R.: Unusual identical cases of ganglioneuroma of the fifth right lumbar root with a clinical picture simulating a discal hernia. Chir Organi Mov 1956; 43: 1-23.

9. Jadro-Santel, D., Grcević, N., Stipancić, I., KrpanAntonin, N.: Lumbar dumbbell ganglioneuroblastoma: a light and electron microscopic study of neuronal degeneration. Neurologija 1988; 37: 49-59.

10. Jeong, G.K., Bendo, J.A.: Lumbar intervertebral disc cyst as a cause of radiculopathy. Spine J 2003; 3: 242-246.

11. Kiyuna, M., Toda, T., Kijima, H., Uechi, H.: A rare case reporto f solitary ganglioneuroma of the transverse colon. Rinsho Byori 2010; 58: 35-38.

12. Kyoshima, K., Sakai, K., Kanaji, M., et al.: Symmetric dumbbell ganglioneuromas of bilateral C2 and C3 roots with intradural extension associated with von Recklinghausen's disease: case report. Surg Neurol 2004; 61: 468-473.

13. Levy, D.I., Bucci, M.N., Weatherbee, L., Chandler, W.F.: Intradural extramedullary ganglioneuroma: case report and review of the literature. Surg Neurol 1992; 37: 216-218.

14. Maitra, A.: Enfermedades de la lactancia y de la infancia. En: Robbins y Cotran. Patología estructural y funcional

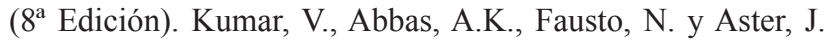
(Eds.). Elsevier. Barcelona, 2010; pp: 447-483.

15. Minkin, K., Tzekov, C., Naydenov, E., et al.: Cerebellar gangliocytoma presenting with hemifacial spasms: clinical report, literature review and possible mechanisms, Acta Neurochir (Wien) 2008; 150: 719-724.

16. Miyakoshi, N., Hongo, M., Kasukawa, Y., Misawa, A., Shimada, Y.: Bilateral and symmetric C1-C2 dumbbell ganglioneuromas associated with neurofibromatosis type 1 causing severe spinal cord compression. Spine J 2010; 10: e11-15.

17. Okamatsu, C. London, W.B., Naranjo, A., et al.: Clinicopathological characteristics of ganglioneuroma and ganglioneuroblastoma: a report from the CCG and COG. Pediatr Blood Cancer 2009; 53: 563-569.

18. Oro, J.J., Geise, A.W.: Dumbbell ganglioneuroma of the lumbar spine associated with a herniated intervertebral disc: case report. Neurosurgery 1983; 13: 711-714.

19. Orborn, A. G.: Schwannoma espinal. En: BrantZawadzki. Los cien diagnósticos principales en columna vertebral. Madrid; Elsevier, 2006; pp: 212-214.

20. Ozawa, H., Kokubun, S., Aizawa, T., Hoshikawa, T., Kawahara, C.: Spinal dumbbell tumors: an analysis of a series of 118 cases. J Neurosurg Spine 2007; 7: 587-593.

21. Pang, B.C., Tchoyoson-Lim, C.C., Tan, K.K.: Giant spinal ganglioneuroma. J Clin Neurosci 2005; 12: 967-972.

22. Papavramidis, T.S., Michalopoulos, N., Georgia, K., et al.: Retroperitoneal ganglioneuroma in an adult patient: a case report and literature review of the last decade. South Med J 
2009; 102: 1065-1067.

23. Peuchmaur, M.: Peripheral neuroblastic tumors: anatomopathological classification. Ann Pathol 2004; 24: 556-567.

24. Radulovi, D.V., Branislav, D., Skender-Gazibara, M.K., Igor, N.M.: Cervical dumbbell ganglioneuroma producing spinal cord compression. Neurol India. 2005; 53:370371 .

25. Saruhashi, Y., Omura, K.; Miyamoto, K.; Katsuura, K.; Hukuda, S.: A migrated lumbar disk herniation simulating a dumbbell tumor. J Spinal Disord 1999; 12: 307-309.

26. Viard, H., Sautreaux, J.L., Haas, O., et al.: Tumeurs nerveuses en sablier. Chirugie 1989; 115: 521-525.

27. Yam, B. Walczyk, K., Mohanty, S.K., Coren, C.V., Katz, D.S.: Radiology-pathology conference: incidental pos-
2011; 22: 434-438

terior mediastinal ganglioneuroma. Clon Imaging 2009; 33: 390-394.

28. Zhang, W.Q., Liu, J.F., Zhao, J., Zhao, S.Y., Xue, Y.: Tumor with watery diarrhoea, hypokalaemia in a 3-year-old girl. Eur J Pediatr 2009; 168: 859-862.

Martínez-Quiñones, J.V.; Consolini, F.; Aso-Escario, J.; Domínguez-Páez, M.; Arregui, R.: Ganglioneuroma coincidente con una extrusión discal lumbar. Neurocirugía 2011; 22: 434-438.

Correspondencia: J.V. Martínez Quiñones. Servicio de Neurocirugía. Hospital MAZ. Avda. Academia General Militar 74. Zaragoza. jvmartínez@maz.es 